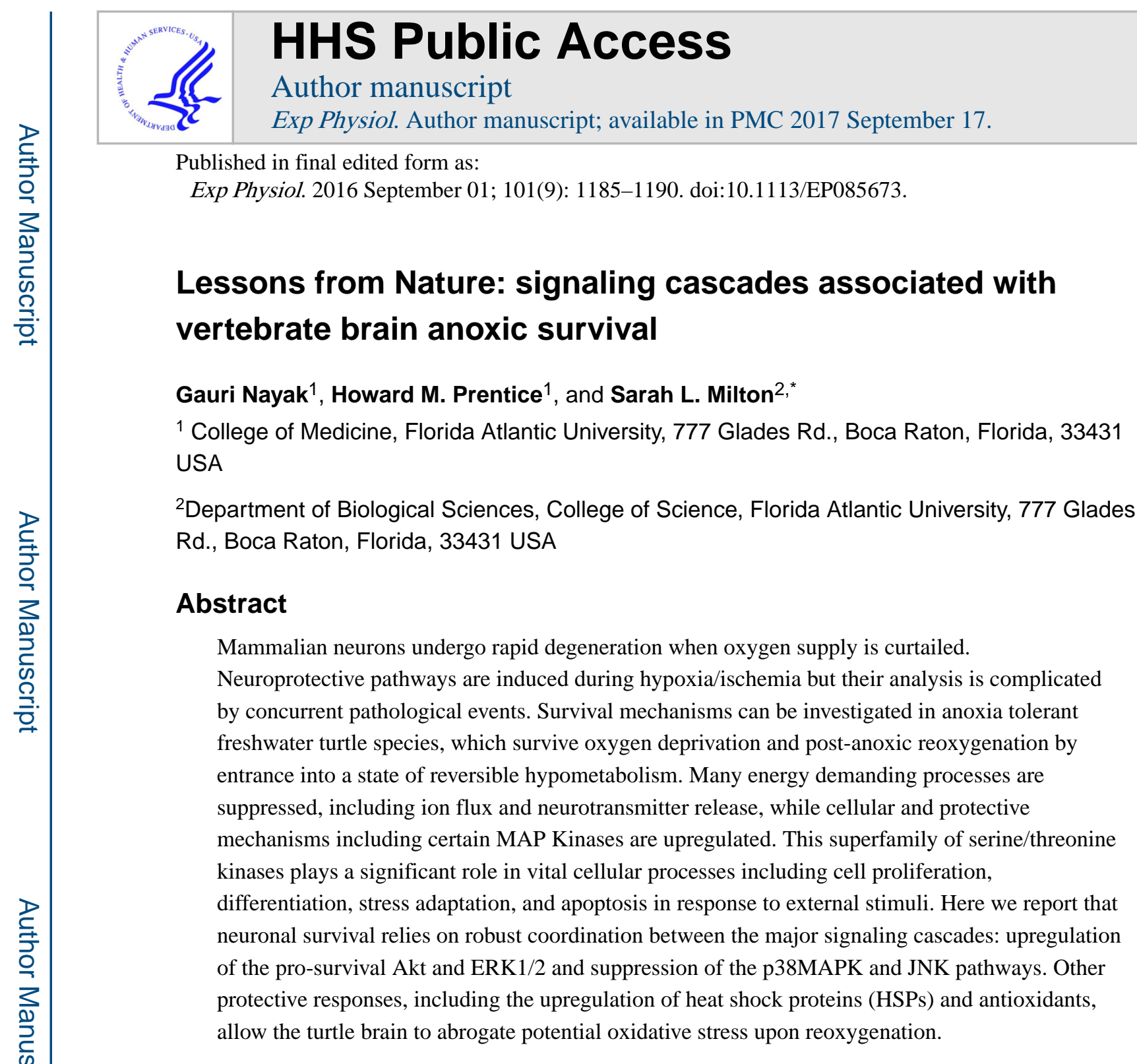

Keywords

Trachemys scripta; anoxia; MAPK

\title{
Introduction
}

While the mammalian brain exhibits an exquisite sensitivity to low oxygen, some vertebrates are able to survive extended periods of hypoxia, while others are able to live for hours to days without oxygen. Animals may encounter low oxygen either acutely (exercise, diving), or chronically (hibernation, burrowing, high altitudes), but a few extremophiles have developed elaborate cerebral defense mechanisms to survive complete anoxia. The most

\footnotetext{
*Corresponding author, Department of Biological Sciences, College of Science, Florida Atlantic University, 777 Glades Rd., Boca Raton, Florida, 33431 USA, Phone: 561-297-3327, Fax: 561-297-2749, smilton@ fau.edu.

Author Contributions: This work was performed in the laboratories of SLM and HP. Experiments were designed by GN, HP, and SLM. Data acquisition and analysis was performed by GN. The ms was written by GN and SLM and reviewed for critical content by SLM and HP. All authors approved the final ms and agree to be accountable for all aspects of the work in ensuring that questions related to the accuracy or integrity of any part of the work are appropriately investigated and resolved.
} 
anoxia tolerant vertebrates include certain species of North American pond turtles (Chrysemys picta and Trachemys scripta), and the crucian carp (Carassius carassius) of northern Europe; these can go for days without oxygen at room temperature and live weeks to months at $3^{\circ} \mathrm{C}$ (Milton \& Prentice, 2007). Survival is the result of complex physiological and molecular adaptations that defend the turtle against the stress of oxygen deprivation, with the suppression of pathological processes and the robust expression of protective mechanisms. These changes occur in three phases in the turtle: an initial inhibition to a highly depressed metabolic state, the long term maintenance of brain function in the absence of oxygen, and recovery in the face of potential oxidative stress (Lutz \& Milton, 2004). Metabolic changes occur against a backdrop of "constitutive preconditioning"; many protective mechanisms are seen even in normoxia, providing ready protection in the face of low oxygen (Prentice et al., 2004; Kesaraju et al., 2014).

Early anoxia is characterized by increases in adenosine and cerebral blood flow, and the suppression of energy intensive processes such as excitatory neurotransmitter release, RNA transcription, and protein synthesis. Decreased ion permeability (channel arrest), and the suppression of action potentials (spike arrest) also decrease energy requirements (Pamenter \& Buck, 2008; Pamenter et al., 2012); together, the reductions in ion flow and neurotransmitter release result in a reversible "coma" (Fernandes et al., 1997). This abrogated electrical activity is accompanied by increases in protective MAPK and heat shock proteins (HSPs) that tilt the balance in favor of cell survival and away from apoptosis (Kesaraju et al., 2009; Nayak et al., 2011). The maintenance of long term anoxia, interestingly, allows for the continued release and reuptake of neurotransmitters, albeit at greatly reduced rates (Milton et al., 2002; Milton \& Lutz, 2005). Many neuroprotective pathways remain activated, and antioxidant levels may even increase. In contrast to mammalian survival mechanisms, cellular protection does not appear to be related to a strong HIF response (Milton and Kesaraju, unpublished data).

\section{The MAP Kinases}

We utilized neuronally enriched primary cultures derived from $T$. scripta to pharmacologically manipulate potentially significant molecular events previously reported to occur in the anoxic turtle in vivo (Milton et al., 2008; Nayak et al., 2011). For this study, cell cultures were exposed to specific MAPK inhibitors prior to normoxia or $4 \mathrm{~h}$ anoxia; small molecule inhibitors were used to block activation of phosphatidylinositol -3-kinase (PI3K/ Akt), extracellular regulated kinase (ERK1/2), pJNK or pp38MAPK (Table I). The concentrations used were determined by a dose dependent titration to impact the pathway of interest without significantly altering other MAPK pathways. Results indicate that survival relies on a robust coordination between the major MAP kinase signaling cascades; turtle neurons utilize an upregulation of the pro-survival pathways pAKT and pERK1/2 and the suppression of the pro-apoptotic MAP kinases p38MAPK and JNK.

\section{Akt}

Phosphorylation of PI3K/Akt occurs in response to a variety of stressors and is generally described as anti-apoptotic. Four hours anoxia increased P-Akt to $186 \pm 10 \%$ of normoxic 
levels; blocking the PI3K pathway decreased p-Akt expression in normoxic and 4h anoxic cultures by $57 \%$ and $87 \%$, respectively, vs. untreated cells (Fig.1). Levels of nonphosphorylated Akt also increased, to $150 \%$ of basal at $4 \mathrm{~h}$ anoxia. Suppression of p-Akt was accompanied by mean increases in phosphorylated p38MAPK and JNK of $25 \%$ above their basal anoxic levels; phospho-ERK levels were not affected by p-Akt suppression.

We previously demonstrated that the mitogen activated protein kinase (MAPK) superfamily modifies cellular responses by altering gene expression and modifying proteins in response to a variety of external stimuli, and are modulated in the anoxic turtle both in vivo (Milton et al., 2008) and in vitro (Nayak et al., 2011). However, a direct role for the various MAPKs in promoting cell survival or cell death in extended anoxia has not been previously demonstrated in this model. We used pathway-specific pharmacological blockers for ERK, JNK, and p38MAPK activation to determine the impact of each pathway on cell survival, with concentrations titrated to change cell survival by $\sim 50 \%$ during anoxia. As in an earlier report (Nayak et al., 2011), all three pathways were activated in vitro over $4 \mathrm{~h}$ anoxia, but with p-ERK levels increasing to a greater degree than p-JNK or p-p38MAPK (Fig.2). Blockade reduced the activation of p-ERK to $72 \%$ of basal in normoxic cultures and in anoxia to $17 \%$ of untreated controls; blockade of the JNK and p38 pathways reduced levels to $36 \%$ and $17 \%$, respectively, of anoxic controls. There was no significant change in nonphosphorylated forms of the MAPKs in anoxia (results not shown).

The upregulation of p-Akt and p-ERK clearly provide protection to the anoxic turtle neuron: pharmacological blockade of either p-Akt or p-ERK increased cell death in a concentration dependent manner (the approximate $\mathrm{LC}_{50}$ 's were utilized in the Western blotting experiments above and in Table I). Conversely, blockade of pJNK or pp38MAPK decreased cell death (values that decreased cell death by $\sim 50 \%$ were used to examine changes to the MAPK pathways described above and in Table I).

In mammalian models, a rapid upregulation of ERK appears to protect ischemic cells if the activation is transient ( $\mathrm{Li}$ et al., 2002), whereas long term ERK activity has been found to increase cell death (Wang et al., 2003). In the anoxic turtle in vivo, ERK is upregulated in the first hour of anoxia (Milton et al., 2008) but then declines, whereas in cell culture ERK remains elevated even over 12h anoxia (Nayak et al., 2011), thus leaving open whether the anoxic cells survive despite ERK upregulation due to the activation of other pro-survival pathways, or if extended ERK activation is instead a symptom of accumulating cell stress that results eventually in death. The activation of JNK and p38MAPK are less controversial, and are generally considered to be pro-apoptotic, though some studies have reported prosurvival effects. As has been reported in numerous studies, there is clearly a great deal of cross talk between members of the MAPK family. The greater cell death when ERK levels are decreased may be related to increases in p-JNK and especially p-p38MAPK, as these were elevated by $47 \%$ and $398 \%$, respectively, over untreated anoxic cells when p-ERK was suppressed. Conversely, blockade of the JNK pathway reduced anoxic cell death by $43 \%$ and was accompanied by increases in p-ERK and p-Akt over anoxic controls of $84 \%$ and $72 \%$, respectively, without changing p-p38MAPK levels. Blockade of the p38MAPK pathway also 
reduced anoxic cell death, and increased p-ERK and p-Akt significantly by a mean $74 \%$ and $51 \%$ over basal anoxia.

It has been proposed that the end mechanism by which Akt and the MAPKs provide neuroprotection is via inhibition of the mitochondrial transition pore that allows Cytochrome c leakage from the mitochondria and thus activation of executioner caspases and apoptosis.

Activated Akt acts to inhibit Bad and caspase-9, and suppresses Cyt c increases in hypoxia/ reoxygenation (reviwed in Hausenloy \& Yellon, 2007); pERK has also been linked to suppression of cyt c release (Li et al., 2002). Along with increases in ERK1/2 and Akt activation we have also reported increased transcription and translation of the major downstream anti-apoptotic protein Bcl-2, compared to the pro-apoptotic Bax (Kesaraju et al., 2014). The greater anoxia-induced increase in Bcl-2 compared to Bax contrasts sharply with the mammalian brain, where ATP depletion is associated with decreased Bcl-2 levels and increases in Bax and activated caspase-3. We hypothesize that greater increases in ERK1/2 and Akt activation, and increased transcription and translation of Bcl-2 vs. Bax stabilize mitochondrial integrity, preventing apoptosis. Although turtle neurons possess apoptotic machinery, our results strongly suggest the compensatory pro-survival molecular pathways reported here and earlier strongly inhibit execution of the apoptotic pathway and promote neuronal survival.

\section{The Re-oxygenation Phase}

Recent work in my laboratory has also focused on the "Recovery" phase of anoxia; in the mammalian brain subjected to severe hypoxia or ischemia, reperfusion is followed by an overproduction of oxygen free radicals including superoxides, hydrogen peroxide, and hydroxyl radicals (Hashimoto et al., 2003). These reactive oxygen species (ROS) damage cellular constituents including proteins, lipids, and nucleic acids and can result in additional brain damage following the original hypoxic insult. Remarkably, the freshwater turtle recovers from extended anoxia without ROS overproduction due to both constitutively high levels of antioxidants (Rice et al., 1995; Willmore \& Storey, 1997), which continue to increase during anoxia, and the suppression of ROS formation linked in part to Hsp72 (Milton et al., 2007; Pamenter et al., 2007; Kesaraju et al., 2014). One potential antioxidant mechanism in T. scripta is the Methionine Sulfoxide Reductase system (MsrA and MsrB), which is upregulated in the turtle brain during anoxia/reoxygenation. Methionine (Met) is one of the most readily oxidized amino acids and Msr may restore the activity of damaged proteins by the reduction of oxidized Met (Spector et al., 2003; Prentice et al., 2008). Msr has been shown in mammalian models to catalytically scavenge ROS before they damage cellular constituents by the reversible oxidation/reduction of readily available protein Met, and increased cell death and ROS damage occur when Msr levels are reduced (Pal et al., 2007; Salmon et al., 2009). The turtle is the first vertebrate model in which Msr transcript and protein levels are shown to be induced by low oxygen concentration, which makes $T$. scripta radically different from other animal models, and provides a unique opportunity to investigate the function and regulation of this peptide that may play a critical role in protection against oxidative damage (Milton, in prep). 


\section{Acknowledgements}

This work was supported in part by the National Institute of Health AREA grant No. 1 R15 NS048909-01 and the American Heart Association (Florida-Puerto Rico Affiliate) grant 04553 18B to SLM, and the Florida Atlantic University Foundation.

\section{References}

Fernandes JA, Lutz PL, Tannenbaum A, Todorov AT, Liebovitch L, Vertes R. Electroencephalogram activity in the anoxic turtle brain. Am J Physiol. 1997; 273:R911-R919. [PubMed: 9321867]

Hashimoto T, Yonetani M, Nakamura H. Selective brain hypothermia protects against hypoxicischemic injury in newborn rats by reducing hydroxyl radical production. Kobe J Med Sci. 2003; 49:83-91. [PubMed: 14970751]

Hausenloy DJ, Yellon DM. Reperfusion injury salvage kinase signalling: taking a RISK for cardioprotection. Heart Fail Rev. 2007; 12:217-234. [PubMed: 17541822]

Kesaraju S, Nayak G, Prentice HM, Milton SL. Upregulation of Hsp72 mediates anoxia/reoxygenation neuroprotection in the freshwater turtle via modulation of ROS. Brain Res. 2014; 1582:247-256. [PubMed: 25107858]

Kesaraju S, Schmidt-Kastner R, Prentice HM, Milton SL. Modulation of stress proteins and apoptotic regulators in the anoxia tolerant turtle brain. J Neurochem. 2009; 109:1413-1426. [PubMed: 19476552]

Li F, Omori N, Sato K, Jin G, Nagano I, Manabe Y, Shoji M, Abe K. Coordinate expression of survival p-ERK and proapoptotic cytochrome $\mathrm{c}$ signals in rat brain neurons after transient MCAO. Brain Res. 2002; 958:83-88. [PubMed: 12468032]

Lutz PL, Milton SL. Negotiating brain anoxia survival in the turtle. J Exp Biol. 2004; 207:3141-3147. [PubMed: 15299035]

Milton SL, Dirk LJ, Kara LF, Prentice HM. Adenosine modulates ERK1/2, PI3K/Akt, and p38MAPK activation in the brain of the anoxia-tolerant turtle Trachemys scripta. J Cereb Blood Flow Metab. 2008; 28:1469-1477. [PubMed: 18506199]

Milton SL, Lutz PL. Adenosine and ATP-sensitive potassium channels modulate dopamine release in the anoxic turtle (Trachemys scripta) striatum. Am J Physiol Regul Integr Comp Physiol. 2005; 289:R77-R83. [PubMed: 15718391]

Milton SL, Nayak G, Kesaraju S, Kara L, Prentice HM. Suppression of reactive oxygen species production enhances neuronal survival in vitro and in vivo in the anoxia-tolerant turtle Trachemys scripta. J Neurochem. 2007; 101:993-1001. [PubMed: 17326763]

Milton SL, Prentice HM. Beyond anoxia: the physiology of metabolic downregulation and recovery in the anoxia-tolerant turtle. Comp Biochem Physiol A Mol Integr Physiol. 2007; 147:277-290. [PubMed: 17049896]

Milton SL, Thompson JW, Lutz PL. Mechanisms for maintaining extracellular glutamate levels in the anoxic turtle striatum. Am J Physiol Regul Integr Comp Physiol. 2002; 282:R1317-R1323. [PubMed: 11959671]

Nayak GH, Prentice HM, Milton SL. Neuroprotective signaling pathways are modulated by adenosine in the anoxia tolerant turtle. J Cereb Blood Flow Metab. 2011; 31:467-475. [PubMed: 20648037]

Pal R, Oien DB, Ersen FY, Moskovitz J. Elevated levels of brain-pathologies associated with neurodegenerative diseases in the methionine sulfoxide reductase A knockout mouse. Exp Brain Res. 2007; 180:765-774. [PubMed: 17333008]

Pamenter ME, Buck LT. Neuronal membrane potential is mildly depolarized in the anoxic turtle cortex. Comp Biochem Physiol A Mol Integr Physiol. 2008; 150:410-414. [PubMed: 18519169]

Pamenter ME, Hogg DW, Gu XQ, Buck LT, Haddad GG. Painted turtle cortex is resistant to an in vitro mimic of the ischemic mammalian penumbra. J Cereb Blood Flow Metab. 2012; 32:2033-2043. [PubMed: 22805876]

Pamenter ME, Richards MD, Buck LT. Anoxia-induced changes in reactive oxygen species and cyclic nucleotides in the painted turtle. J Comp Physiol B. 2007; 177:473-481. [PubMed: 17347830] 
Prentice HM, Milton SL, Scheurle D, Lutz PL. The upregulation of cognate and inducible heat shock proteins in the anoxic turtle brain. J Cereb Blood Flow Metab. 2004; 24:826-828. [PubMed: 15241191]

Prentice HM, Moench IA, Rickaway ZT, Dougherty CJ, Webster KA, Weissbach H. MsrA protects cardiac myocytes against hypoxia/reoxygenation induced cell death. Biochem Biophys Res Commun. 2008; 366:775-778. [PubMed: 18083115]

Rice ME, Lee EJ, Choy Y. High levels of ascorbic acid, not glutathione, in the CNS of anoxia-tolerant reptiles contrasted with levels in anoxia-intolerant species. J Neurochem. 1995; 64:1790-1799. [PubMed: 7891107]

Salmon AB, Pérez VI, Bokov A, Jernigan A, Kim G, Zhao H, Levine RL, Richardson A. Lack of methionine sulfoxide reductase $\mathrm{A}$ in mice increases sensitivity to oxidative stress but does not diminish life span. FASEB J. 2009; 23:3601-3608. [PubMed: 19487311]

Spector D, Etienne F, Brot N, Weissbach H. New membrane-associated and soluble peptide methionine sulfoxide reductases in Escherichia coli. Biochem Biophys Res Commun. 2003; 302:284-289. [PubMed: 12604343]

Wang Z, Chen X, Zhou L, Wu D, Che X, Yang G. Effects of extracellular signal-regulated kinase (ERK) on focal cerebral ischemia. Chin Med J (Engl). 2003; 116:1497-1503. [PubMed: 14570609]

Willmore WG, Storey KB. Glutathione systems and anoxia tolerance in turtles. Am J Physiol. 1997; 273:R219-R225. [PubMed: 9249553] 


\section{New Findings}

\section{What is the topic of this review?}

While the mammalian brain is exquisitely sensitive to hypoxia, some turtles survive complete anoxia by decreasing metabolic demand to match reduced energy supply. These alternative animal models may help elucidate neuroprotective mechanisms and reveal novel therapeutic targets for diseases of oxygen deprivation.

\section{What advances does it highlight?}

The MAPKs are part of the suite of adaptive responses to anoxia that are modulated by adenosine, a "retaliatory metabolite" released in early anoxia. In anoxic turtle neurons, upregulation of pro-survival Akt and ERK1/2 and suppression of the p38MAPK and JNK pathways promote cell survival, as does the anoxic- and post-anoxic upregulation of the antioxidant methionine sulfoxide reductase. 
A
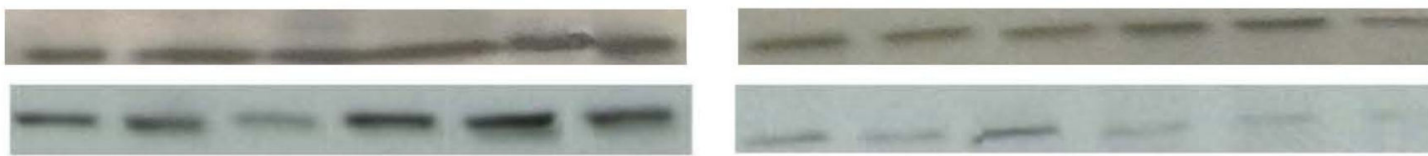

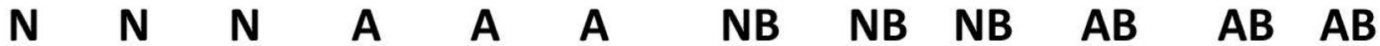

B

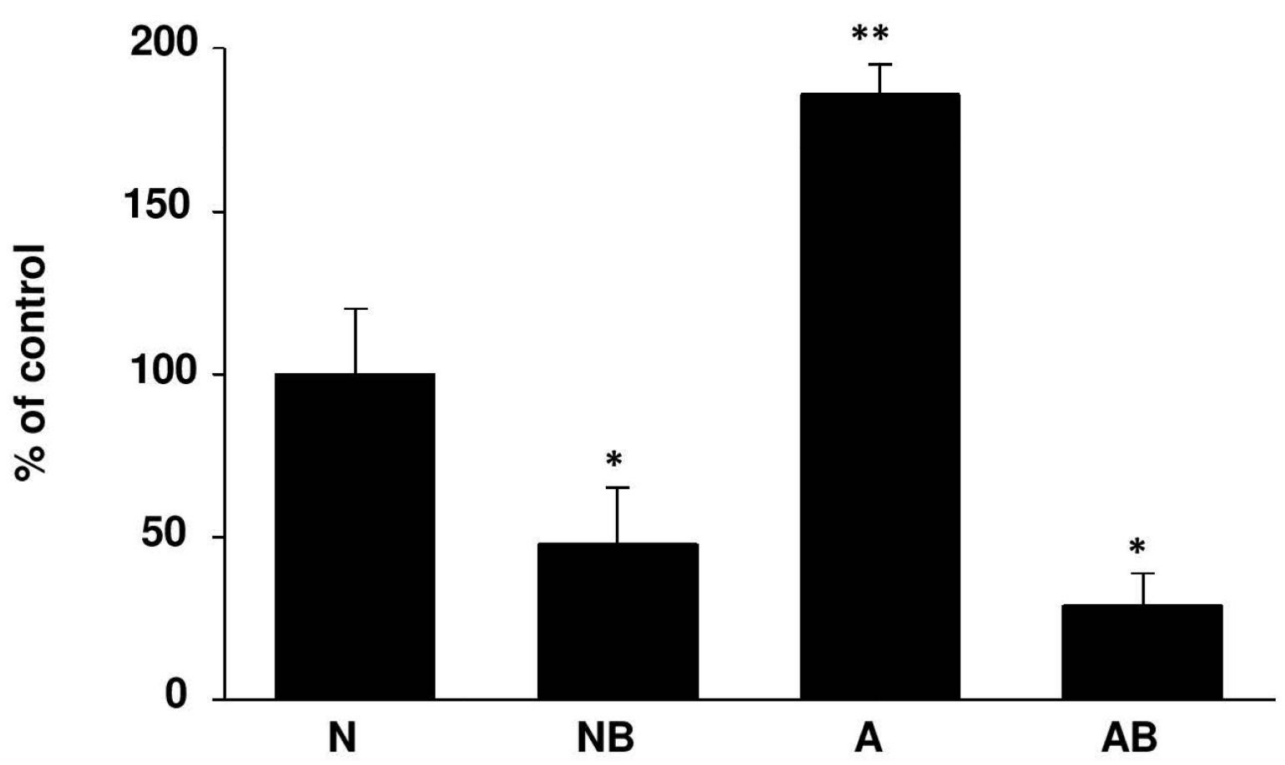

Figure 1.

Phosphorylated Akt increases over $4 \mathrm{~h}$ anoxia in cultures of primary turtle neurons; increases are abrogated by the specific PI3K pathway inhibitor LY294002 (40 $\mu \mathrm{M})$. (A) Representative western blot of changes in p-Akt, with or without PI3K inhibition. Actin does not change with anoxia/reoxygenation in this model (Kesaraju et al., 2014) (B) Densitometry analysis of changes in p-Akt. Data shown as mean \pm SEM. $* *=$ significantly different from normoxic controls, $*$ = significantly different from untreated controls at same timepoint . Data are normalized to actin signal.. $\mathrm{N}=$ normoxic controls, $\mathrm{NB}=$ normoxic block, $A=4 \mathrm{~h}$ anoxia, $\mathrm{AB}=4 \mathrm{~h}$ anoxic block. Statistically significant differences between treatments were evaluated by ANOVA with post-hoc analysis using the SPSS statistical package. Differences were considered statistically significant at $\mathrm{p}<0.05, \mathrm{~N}=5-6$. 
A

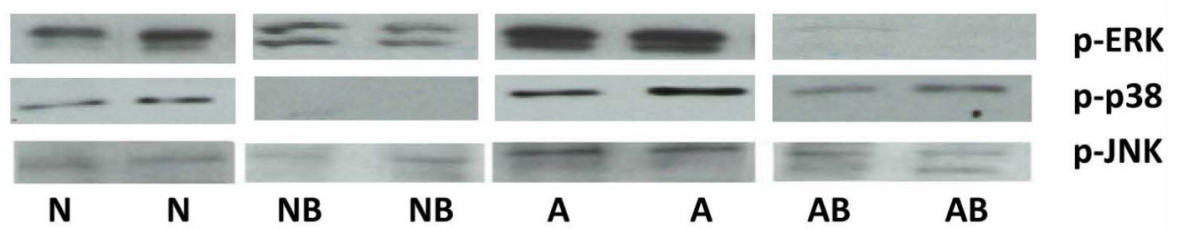

B
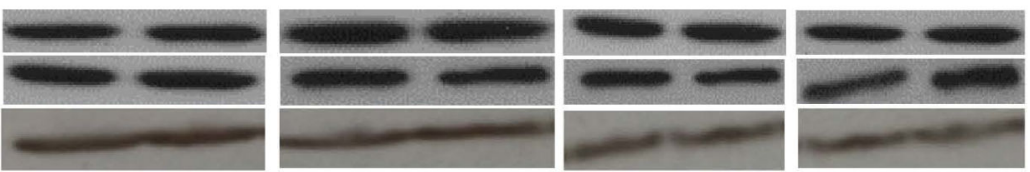

p-ERK p-p38

$\mathbf{N} \quad \mathbf{N}$

NB

NB

A

A

$A B$

p-JNK

C

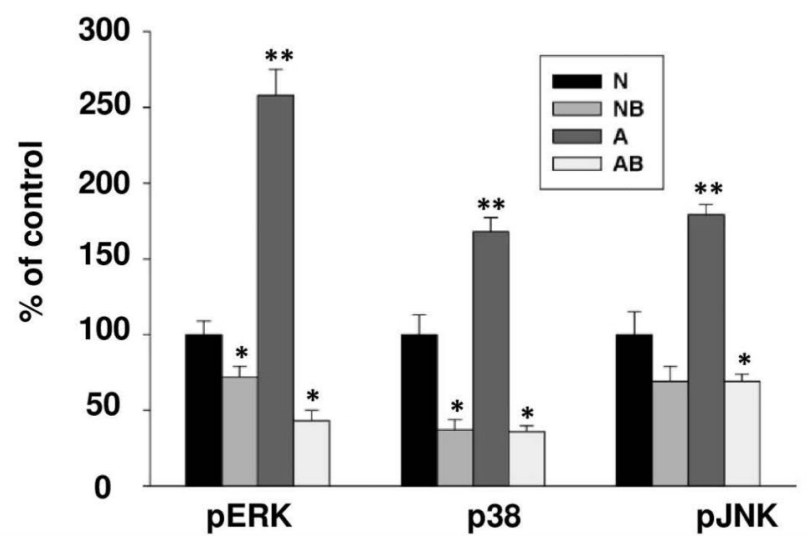

Figure 2.

Phosphorylated MAPKs increase over $4 \mathrm{~h}$ anoxia in cultures of primary turtle neurons; increases are abrogated by specific pathway blockers. Representative western blots of MAPKs (A) and actin (B). Densitometric analysis (C) showing anoxia-induced activation of MAPKs abrogated by their respective specific blockers: ERK, U0126 (5.0 $\mu \mathrm{M})$; JNK SP600125 $(5.0 \mu \mathrm{M})$; p38MAPK SB203580 $(1.0 \mu \mathrm{M})$. Data shown as mean \pm SEM. $* *=$ significantly different from normoxic controls, $*$ = significantly different from untreated controls at same timepoint, $\mathrm{p}<0.05 . \mathrm{N}=$ normoxic controls, $\mathrm{NB}=$ normoxic block, $\mathrm{A}=4 \mathrm{~h}$ anoxia, $A B=4 \mathrm{~h}$ anoxic block. Data are normalized to actin signal. Actin does not change with anoxia/reoxygenation in this model (Fig. part A). Statistically significant differences between treatments were evaluated by ANOVA with post-hoc analysis using the SPSS statistical package. Differences were considered statistically significant at $\mathrm{p}<0.05, \mathrm{~N}=5$ individuals/treatment. 


\section{Table I}

PI3K/Akt and MAPK blockers significantly alter cell death, with pAkt and pERK blockade increasing cell death and pJNK and pp38MAPK inhibition decreasing cell death.

\begin{tabular}{|c|c|c|}
\hline & \multicolumn{2}{|c|}{$\%$ cell death $($ mean $\pm \mathrm{SEM})$} \\
\hline Pathway & Control (DMSO) & $\begin{array}{c}\text { Pharmacological } \\
\text { blockade }\end{array}$ \\
\hline \multicolumn{3}{|c|}{ Akt blocker (LY294002, $40 \mu \mathrm{M})$} \\
\hline Normoxia & $7 \pm 2$ & $15 \pm 1$ \\
\hline $4 \mathrm{hr}$ anoxia & $20 \pm 1^{l}$ & $56 \pm 4^{1,2}$ \\
\hline \multicolumn{3}{|c|}{ ERK blocker (U0126, $5.0 \mu \mathrm{M}$ ) } \\
\hline Normoxia & $12 \pm 2$ & $16 \pm 2$ \\
\hline $4 \mathrm{hr}$ anoxia & $18 \pm 3$ & $40 \pm 1^{1,2}$ \\
\hline \multicolumn{3}{|c|}{ JNK blocker (SP600125, 5.0 $\mu \mathrm{M}$ ) } \\
\hline Normoxia & $7 \pm 2$ & $12 \pm 4$ \\
\hline $4 \mathrm{hr}$ anoxia & $23 \pm 2^{1}$ & $13 \pm 1^{2}$ \\
\hline \multicolumn{3}{|c|}{ 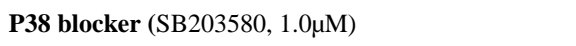 } \\
\hline Normoxia & $7 \pm 2$ & $10 \pm 3$ \\
\hline $4 \mathrm{hr}$ anoxia & $23 \pm 3^{1}$ & $14 \pm 1^{1,2}$ \\
\hline
\end{tabular}

Carrier DMSO alone does not significantly increase cell death above non-DMSO controls (7-10\%). Superscripts:

${ }^{1}=$ significantly different from normoxia, same treatment;

${ }^{2}=$ significantly different from anoxic controls, $\mathrm{p}<0.05, \mathrm{~N}=4-5$ individuals per group. 